\title{
DATOS PARA LA HISTORIA DE LA MALACOLOGIA ESPAÑOLA: LAS EDICIONES DEL CATALOGO DE GRAELLS
}

\section{Rodolfo Gozalo Fernando Robles}

El Catálogo de los Moluscos terrestres y de agua dulce observados en España, y descripción y notas de algunas especies nuevás ó poco conocidas del mismo país (en adelante, Catálogo), realizado por Mariano de la Paz Graells y al que se le asigna la fecha de 1846, posee un triple interés. Por una parte es la primera (¡y única todavía!) síntesis sobre el tema que abarca el conjunto de la España peninsular y de las Islas Baleares. Én segundo lugar, constituye el primer trabajo malacológico realizado por un autor español. Por último, aunque en àlgunos aspectos es lo más importante, se describen en él varias especies nuevas, algunas de las cuales han sido incluídas en la sinonimia de especies más antiguas o han cambiado de nombre, mientras que otras conservan validez plena (véase apéndice).

Recientemente Mienis (1992) señala la existencia de dos ejemplares de esta obra, depositados en la Biblioteca de la Colección de Moluscos de la Universidad Hebrea de Jerusalén, que incorporan láminas distintas que se diferencian por el tamaño, la disposición de las figuras y algunos 
aspectos del dibujo de éstas. Mienis indica que el texto y tamaño de las restantes páginas de los dos ejemplares son idénticos y plantea el problema de cuál de las dos láminas es la original.

La existencia de estas dos láminas en ejemplares del Catálogo de Graells fue puesta ya de manifiesto por Azpeitia.(1929). Además, este autor cita una segunda edición de esta obra, sin fecha ni pie de imprenta, en la que se incluye la más pequeña de las dos láminas y que contiene además algunas modificaciones respecto al texto de la primera edición. Por último, comenta que «He visto algún ejemplar con el texto de la primera edición y la lámina de la segunda", lo que concuerda con lo observado por Mienis (o. c.) y resuelve, al menos aparentemente, el tema de la prioridad: la lámina original sería la de mayor tamaño $(18 \times 26 \mathrm{~cm})$ y la más pequeña $(12 \times 19,2 \mathrm{~cm})$ se realizaría para acompañar a la segunda edición, adicionándose a algunos ejemplares del texto de la primera edición, que aparentemente carecerían de la lámina original.

Robles (1975) aclara la fecha de esta segunda edición, que en realidad se publicó como un apéndice, con paginación independiente, de la segunda edición corregida del Tratado completo de Historia Natural (en adelante, Tratado), obra original del autor francés Apollinaire Bouchardat, traducida por Luis Sánchez Toca e impresa en Madrid en la imprenta de Hilario Martínez en 1848. Esto explica que la segunda edición del Catálogo carezca de fecha y de pie de imprenta, que ya figuran en la portada del Tratado de Bouchardat, así como justifica la escasez de ejemplares de dicha segunda edición del Catálogo, que ha pasado desapercibida hasta el extremo de que Azpeitia (o. c.) señala que «esta segunda edición es muy poco conocida y resulta una verdadera rareza entre los bibliófilos, hasta tal punto, que no la he encontrado en ninguna de las Bibliotecas que he consultado y no conozco más ejemplar que el que yo poseo, debiéndoselo a la amabilidad de la familia del autor». Sin embargo este desconocimiento no deja de ser paradójico, ya que el Tratado de Bouchardat, del que constituía un apéndice como hemos indicado, fue uno de los primeros textos de Ciencias Naturales de uso en la Segunda Enseñanza española (Sos, 1988) y debió de tener una gran difusión, como lo demuestra el que se realizasen dos ediciones en años consecutivos (1847 y 1848). En realidad los ejemplares de esta segunda edición del Catálogo no eran tan raros, ya que figura en la bibliografía de Haas (1929) (aunque con fecha errónea: 1846) y, según el propio Azpeitia (o. c.), es citada por Kennard y Woodward (1926). 
Aclarado pues el tema de las dos láminas distintas y de la existencia, al menos, de dos ediciones de la obra de Graells, se plantea, no obstante, un segundo problema, relacionado con la fecha de impresión de su primera edición, que se sitúa generalmente en 1846.

La traducción castellana del Tratado de Bouchardat se publicó por primera vez en 1847 e incluía, como adición, la primera edición del Catálogo de Graells (Gomis et al., 1988; Josa, 1992). Este apéndice carece de fecha y pie de imprenta ya que, como en el caso de la segunda edición, que hemos comentado, estos datos figuran en la portada general del volúmen al que se adiciona el Catálogo. Por otra parte, existen ejemplares de la obra de Graells, encuadernados independientemente del Catálogo, que sólo se diferencian por la adición en la portada de una fecha, 1846, y de la indicación de los puntos de venta (Madrid: Librería de los Señores Viuda e Hijos de Don Antonio Calleja y Lima. Casa de los Señores Calleja, Ojea y Compañía). En la página opuesta, detrás de la anteportada, figura la imprenta de H. Martínez, donde se imprimió el libro. Con estas salvedades, el texto y la lámina desplegable de ambas publicaciones son idénticos.

No es posible aclarar, con los datos que poseemos, si Graells escribió y editó su Catálogo independientemente de la traducción y edición del Tratado de Bouchardat y posteriormente se adicionó como un apéndice a esta obra, o si bien fue un encargo del editor de la traducción del Tratado, destinado ya inicialmente a figurar como un apéndice, junto con la $\mathrm{Me}$ moria sobre el modo de hacer las herborizaciones y los herbarios de Miguel Colmeiro, que le acompaña. En el segundo caso, los ejemplares sueltos, con portada propia y fecha de 1846, corresponderían a tiradas aparte, encargadas por Graells, de su apéndice al Tratado de Bouchardat. En cualquier caso, estas separatas fueron adicionadas con una portada en la que figura la fecha de 1846, lo que les asigna prioridad, desde el punto de vista del Código Internacional de Nomenclatura Zoológica, sobre el apéndice al Tratado, que no fue puesto a la venta hasta 1847, según consta en el pie de imprenta de la primera edición. Dado que el manuscrito de Graells fue acabado, según reza en su prólogo, el 24 de enero de 1846 y que el apéndice al Tratado lleva una paginación independiente de éste, no sería de extrañar que se acabase de imprimir durante 1846 mientras que el Tratado, mucho más voluminoso, no se finalizase hasta el año siguiente. Mientras tanto, y a la espera de que saliera a la venta el Tratado, Graells pudo encargar una tirada restringida de su Catálogo, fecharla en 1846 y distribuirla entre sus corresponsales, costumbre frecuente en la época. A 
favor de esta interpretación podemos aducir dos argumentos. En primer lugar, el formato y tipografía del Catálogo son idénticos a los del Tratado, por lo que parece que fueron concebidos para editarse conjuntamente. En segundo lugar, y como argumento de mayor peso, hay que señalar que en la portada de la edición corregida del Catálogo, adicionada a la segunda edición del Tratado de Bouchardat (1848) figura, debajo del nombre del autor, la indicación de "segunda edición", mientras que el apéndice a la primera edición de la traducción del Tratado (Bouchardat, 1847) no presenta ninguna indicación en este sentido, lo que hace suponer que Graells la consideraba como la primera edición de su obra.

En cualquier caso, y como conclusión, podemos considerar, a efectos de prioridad en la nomenclatura, la existencia de las siguientes ediciones del Catálogo de Graells: Primera edición: 1846. Corresponden a ella los ejemplares sueltos con portada propia y fecha 1846, dotados de lámina grande plegada. Existen ejemplares, al menos, en la Biblioteca del Museo Nacional de Ciencias Naturales de Madrid (C.S.I.C.) y en la Biblioteca de la Colección de Moluscos de la Universidad Hebrea de Jerusalén (Israel) (Mienis, 1992).

Primera edición, primera reimpresión: 1847. Corresponde a los ejemplares del catálogo adicionado a la primera edición de la traducción castellana del Tratado completo de Historia Natural, de A. Bouchardat.

Segunda edición, corregida: 1848. Corresponde a los ejemplares adicionados a la segunda edición de dicho Tratado y en su portada figura la indicación «segunda edición».

Existen además ejemplares de la primera edición que incorporan la lámina de la segunda edición (Azpeitia, 1929; Mienis, 1992) y que, por consiguiente, fueron repartidos a partir de 1848.

Por último, cabe señalar la existencia de una edición facsímil del $\mathrm{Ca}$ tálogo, realizada por el Servicio de Reproducción de Libros de las Librerías «París-Valencia»(Valencia, 1991), a partir del apéndice a la primera edición del Tratado de Bouchardat, de acuerdo con las características de la portada.

\section{Agradecimientos}

Los autores agradecen a la Dra. M. ${ }^{\text {a }}$ Angeles Ramos, del Museo Nacional de Ciencias Naturales (C.S.I.C.), la información suministrada sobre la primera edición del Catálogo de Graells; y al Dr. Francisco Pelayo, del 
Centro de Estudios Históricos (C.S.I.C.), la información sobre la primera edición del Tratado de Bouchardat.

\section{APÉNDICE}

Status actual de los taxones descritos por Graells (1846) en su Catálogo de los Moluscos terrestres y de agua dulce observados en España.

Helix Grateloupi Graells, 1846. La especie es válida pero el nombre estaba pre-ocupado por Helix Grateloupi Pfeiffer, 1842, por lo que fue sustituido por el de Helix graellsiana Pfeiffer, 1853. En la actualidad se utiliza la combinación Allognathus graellsianus (Pfeiffer, 1853) para esta especie.

Cyclostoma Velascoi Graells, 1846. Como indica el propio Graells, es un sinónimo de Cyclostoma Voltzianum Michaud, 1833, a su vez sinónimo posterior de Leonia mamillaris (Lamarck, 1822).

Planorbis Dufourii Graells, 1846. Suele considerarse sinónimo posterior de Planorbarius metidjensis. (Forbes, 1838), aunque serían necesarios estudios anatómicos para confirmar esta sinonimia. Es posible que Planorbarius dufouri (Graells, 1846) sea una especie válida.

Paludina impura Lam. var. matritensis Graells, 1846. Es sinónimo posterior de Bithynia tentaculata (Linnaeus, 1758).

Neritina Velascoi Graells, 1846. Especie válida, actualmente asignada al género Theodoxus.

Neritina valentina Graells, 1846. Sinónimo posterior de Theodoxus velascoi (Graells, 1846).

\section{BIBLIOGRAFIA}

Azpeitia Morós, F. (1929). Monografía de las Melanopsis vivientes y fósiles de España. Mem. Inst. Geol. Min. España. Madrid.

Bouchardat, A. (1847). Tratado completo de Historia Natural, traducido por D. Luis Sánchez Toca y adicionado con un Catálogo de los Moluscos terrestres y de agua dulce de España, por el doctor D. M. P. Graells y con una memoria sobre el modo de hacer las herborizaciones y los herbarios, por don Miguel Colmeiro. Imprenta de Hilario Martínez. Madrid .

BouchaRdaT, A. (1848). Tratado completo de Historia Natural, traducido por D. Luis Sánchez Toca y adicionado con un Catálogo de los Moluscos terrestres y de agua dulce de España, por el doctor D. M. P. Graells, y con una Memoria sobre el modo de hacer las 
herborizaciones y los herbarios, por D. Miguel Colmeiro. Segunda edición corregida. Imprenta de Hilario Martínez. Madrid.

Gomis, A.; Josa, J.; Fernandez, F. y Pelayo, F. (1988). Historia Natural. Catálogo ilustrado siglos XVIII y XIX. Ministerio de Educación y Ciencia. Madrid.

GRAELLS, M. P. (1846). Catálogo de los Moluscos terrestres y de agua dulce observados en España, y descripción y notas de algunas especies nuevas ó poco conocidas del mismo país. Imprenta de H. Martínez. Madrid y Lima.

HaAs, F. (1929), «Fauna malacológica terrestre y de agua dulce de Cataluña». Treb. Mus. Cienc. Nat. Barcelona, 13: 1-491.

JosA LlORCA, J. (1992). «La Historia Natural en la España del siglo XIX: Botánica y Zoología". Ayer, 7: 109-152.

KenNaRD, A. S. y WOODWARD, F. L. S. (1926). Synonymy of the British non marine Mollusca (Recent and Post-tertiary). London.

Mienis, H. K. (1992). «Comentarios sobre la(s) lámina(s) del "Catálogo de los Moluscos terrestres y de agua dulce de España" ", de M. P. Graells, 1846. Noticiario de la Soc. Esp. Malacología, 16: 14-15.

RobLes, F. (1975). «El género Melanopsis Férussac en el Neógeno continental de la cuenca del río Júcar» (1. ${ }^{a}$ nota). R. Soc. Española Nist. Nat., Vol. Extr. Primer Centenario, 1: 357-368.

Sos BAYNAT, V. (1988). «Sobre la enseñanza de la Geología general en el Bachillerato de España (1845-1936)». Bol. Inst. Libre Enseñanza, 5: 3-12. 